\title{
SGLT2 Inhibitor, Canagliflozin, Attenuates Myocardial Infarction in the Diabetic and Nondiabetic Heart
}

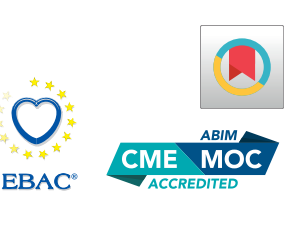

Ven G. Lim, MBCнB, ${ }^{\mathrm{a}, *}$ Robert M. Bell, $\mathrm{PHD},{ }^{\mathrm{a}, *}$ Sapna Arjun, $\mathrm{PHD},{ }^{\mathrm{a}}$ Maria Kolatsi-Joannou, PHD, ${ }^{\mathrm{b}}$ David A. Long, PHD, Derek M. Yellon, $\mathrm{PHD}, \mathrm{DSc}^{\mathrm{a}}$

\section{JACC: BASIC TO TRANSLATIONAL SCIENCE CME/MOC/ECME}

This article has been selected as this month's JACBTS CME/MOC/ECME activity, available online at http://www.acc.org/jacc-journals-cme by selecting the JACC Journals CME/MOC/ECME tab.

Accreditation and Designation Statement

The American College of Cardiology Foundation (ACCF) is accredited by the Accreditation Council for Continuing Medical Education (ACCME) and the European Board for Accreditation in Cardiology (EBAC) to provide continuing medical education for physicians.

The ACCF designates this Journal-based CME/MOC/ECME activity for a maximum of 1 AMAPRA Category 1 Credit or 1 EBAC Credit. Physicians should only claim credit commensurate with the extent of their participation in the activity.

Successful completion of this CME activity, which includes participation in the evaluation component, enables the participant to earn up to 1 Medical Knowledge MOC point in the American Board of Internal Medicine's (ABIM) Maintenance of Certification (MOC) program. Participants will earn MOC points equivalent to the amount of CME credits claimed for the activity. It is the CME activity provider's responsibility to submit participant completion information to ACCME for the purpose of granting ABIM MOC credit.

SGLT2 Inhibitor, Canagliflozin, Attenuates Myocardial Infarction in the Diabetic and Nondiabetic Heart will be accredited by the European Board for Accreditation in Cardiology (EBAC) for 1 hour of External CME credits. Each participant should claim only those hours of credit that have actually been spent in the educational activity. The Accreditation Council for Continuing Medical Education (ACCME) and the European Board for Accreditation in Cardiology (EBAC) have recognized each other's accreditation systems as substantially equivalent. Apply for credit through the post-course evaluation.

Method of Participation and Receipt of CME/MOC/ECME Certificate

To obtain credit for JACBTS: Basic to Translational Science CME/MOC/ECME, you must:

1. Be an ACC member or JACBTS subscriber.

2. Carefully read the CME/MOC/ECME-designated article available online and in this issue of the journal.

3. Answer the post-test questions. At least 2 questions provided must be answered correctly to obtain credit.

4. Complete a brief evaluation.
5. Claim your CME/MOC/ECME credit and receive your certificate electronically by following the instructions given at the conclusion of the activity.

CME/MOC/ECME Objective for This Article: Upon completion of this activity, the learner should be able to: 1) discuss the effects of treatment with canagliflozin on myocardial infarct size among diabetic ZDF and non-diabetic ZL rats; 2) examine the guideline recommendations for prevention of acute myocardial infarction in patients with diabetes mellitus; and 3) define the potential applications of canagliflozin on cardiovascular health among people with or at risk for cardiovascular disease.

CME/MOC/ECME Editor Disclosure: CME/MOC/ECME Editor L. Kristin Newby, MD, is supported by research grants from Amylin, Bristol-Myers Squibb Company, GlaxoSmithKline, Sanofi, Verily Life Sciences (formerly Google Life Sciences), the MURDOCK Study, NIH, and PCORI; receives consultant fees/honoraria from BioKier, DemeRx, MedScape/TheHeart.org, Metanomics, Philips Healthcare, Roche Diagnostics, CMAC Health Education \& Research Institute; serves as an Officer, Director, Trustee, or other fiduciary role for the AstraZeneca HealthCare Foundation and the Society of Chest Pain Centers (now part of ACC); and serves in another role for the American Heart Association and is the Deputy Editor of JACC: Basic to Translational Science.

Author Disclosures: Janssen-Cilag provided funding support for this study and the formulated treatment diets for the long-term oral administration study and the treatment drug for the ex vivo administration study. Dr. Bell is supported by the National Institute of Health (NIHR) University College London Biomedical Research Centre (BRC) and work is supported by the British Heart Foundation (PG/18/10/33550). Dr. Long's laboratory is supported by a Medical Research Council (MR/P018629/1), Diabetes UK (13/ 0004763, 15/0005283), Kidney Research UK (RP36/2015), and by the NIHR BRC at Great Ormond Street Hospital for Children NHS Foundation Trust and University College London. Prof. Yellon has served on a global advisory board for Novo Nordisk. All other authors have reported that they have no relationships relevant to the contents of this paper to disclose.

Medium of Participation: Online (article and quiz).

CME/MOC/ECME Term of Approval

Issue Date: February 2019

Expiration Date: January 31, 2020

From ${ }^{a}$ The Hatter Cardiovascular Institute, University College London, London, United Kingdom; and the ${ }^{\mathrm{b}}$ Developmental Biology and Cancer Programme, UCL Great Ormond Street Institute of Child Health, London, United Kingdom. *Drs. Lim and Bell contributed equally to this work and are joint first authors. Janssen-Cilag provided funding support for this study and the 


\section{SGLT2 Inhibitor, Canagliflozin, Attenuates Myocardial Infarction in the Diabetic and Nondiabetic Heart}

Ven G. Lim, MBCнB, a, aobert M. Bell, PнD,,${ }^{\mathrm{a}, *}$ Sapna Arjun, PнD, ${ }^{\mathrm{a}}$ Maria Kolatsi-Joannou, PнD, ${ }^{\mathrm{b}}$ David A. Long, PнD, Derek M. Yellon, PHD, DSc ${ }^{a}$

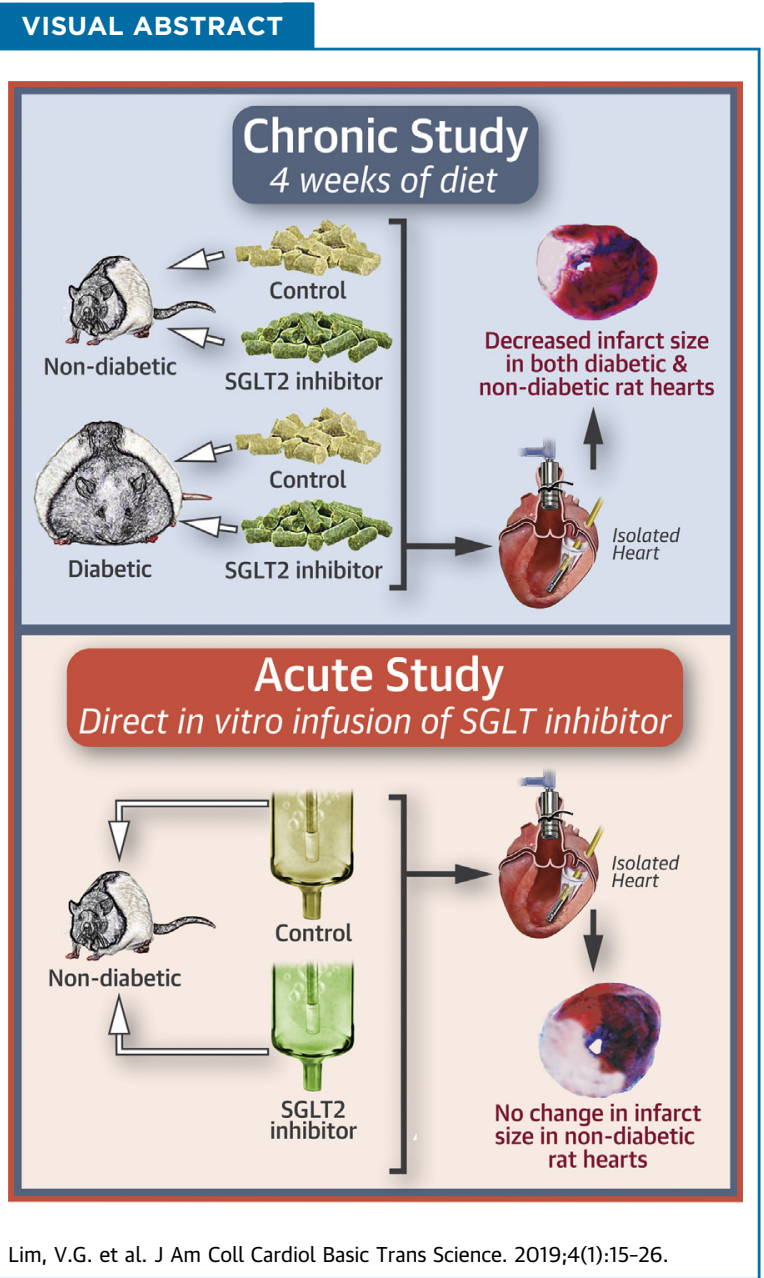

\section{HIGHLIGHTS}

- Long-term SGLT2 inhibition with dietary canagliflozinin diabetic and nondiabetic rats attenuates myocardial ischemia/ reperfusion injury ex vivo.

- This suggests that the improvement in myocardial infarct size by SGLT2 inhibition may occur independent of the glycemic status.

- Canagliflozin improved hyperglycemia in diabetic rats but importantly did not cause hypoglycemia in nondiabetic rats.

- Short-term perfusion of the nondiabetic heart with canagliflozin, solubilized in the Langendorff perfusion buffer, had no impact on the myocardial infarct size.

formulated treatment diets for the long-term oral administration study and the treatment drug for the ex vivo administration study. Dr. Bell is supported by the National Institute of Health (NIHR) University College London Biomedical Research Centre (BRC) and work is supported by the British Heart Foundation (PG/18/10/33550). Dr. Long's laboratory is supported by a Medical Research Council (MR/P018629/1), Diabetes UK (13/0004763, 15/0005283), Kidney Research UK (RP36/2015), and by the NIHR BRC at Great Ormond Street Hospital for Children NHS Foundation Trust and University College London. Prof. Yellon has served on a global advisory board for Novo Nordisk. All other authors have reported that they have no relationships relevant to the contents of this paper to disclose.

All authors attest they are in compliance with human studies committees and animal welfare regulations of the authors' institutions and Food and Drug Administration guidelines, including patient consent where appropriate. For more information, visit the JACC: Basic to Translational Science author instructions page. 


\section{SUMMARY}

The authors hypothesized that despite similar cardiovascular event rates, the improved cardiovascular survival from sodium glucose transporter 2 (SGLT2) inhibition, seen clinically, could be via a direct cytoprotective effect, including protection against myocardial ischemia/reperfusion injury. Langendorff-perfused hearts, from diabetic and nondiabetic rats, fed long-term for 4 weeks with canagliflozin, had lower infarct sizes; this being the first demonstration of canagliflozin's cardioprotective effect against ischemia/reperfusion injury in both diabetic and nondiabetic animals. By contrast, direct treatment of isolated nondiabetic rat hearts with canagliflozin, solubilized in the isolated Langendorff perfusion buffer, had no impact on infarct size. This latter study demonstrates that the infarct-sparing effect of long-term treatment with canagliflozin results from either a glucose-independent effect or up-regulation of cardiac prosurvival pathways. These results further suggest that SGLT2 inhibitors could be repurposed as novel cardioprotective interventions in high-risk cardiovascular patients irrespective of diabetic status. (J Am Coll Cardiol Basic Trans Science 2019;4:15-26) (c) 2019 The Authors. Published by Elsevier on behalf of the American College of Cardiology Foundation. This is an open access article under the CC BY license (http://creativecommons.org/licenses/by/4.0/).
ABBREVIATIONS AND ACRONYMS

DMSO = dimethyl sulfoxide NHE = sodium hydrogen exchange

NS = not significant SGLT2 = sodium glucose transporter 2 ZDF = Zucker Diabetic Fatty ZL = Zucker Lean

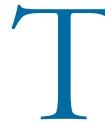
he remarkable cardiovascular benefits of sodium/glucose co-transporter 2 (SGLT2) inhibitors are now well recognized in highrisk type 2 diabetic patients following the landmark clinical trials, EMPA-REG OUTCOME (Empagliflozin Cardiovascular Outcome Event Trial in Type 2 Diabetes Mellitus) (1) and CANVAS (CANagliflozin CardioVAScular Assessment Study) (2), and is further supported by positive outcome data from DECLARETIMI 58 trial (Multicenter Trial to Evaluate the Effect of Dapagliflozin on the Incidence of Cardiovascular Events-Thrombolysis In Myocardial Infarction 58) announced at the recent European Society of Cardiology World Congress. These studies, both designed as noninferiority investigations mandated by the regulatory authorities, revealed an unexpected benefit and superiority over existing standard diabetic care, with a significant reduction of cardiovascular mortality. Equally remarkably, this reduction in cardiovascular mortality was seen notably early-within 1 to 2 months-following the introduction of the respective SGLT2 inhibitor. The mechanism underlying the reduction in cardiovascular mortality is not clear and has been subject to much conjecture: seemingly, improvements in blood sugar control were comparatively minor and improvements in terms of diuresis, weight loss, and blood pressure reduction inadequate to fully explain the differences observed. Indeed, many, including ourselves, have speculated a potential pleiotropic beneficial effect for this class of glucose-lowering therapy (3-5).

The hypothesis that SGLT2 inhibitors may have pleiotropic effects appears to be supported by other observations from the clinical trial data, not least that SGLT2 inhibition appears to have minimal impact upon the cardiovascular event rate-be it myocardial infarction or stroke, admissions with unstable angina or the need for a coronary revascularization procedure $(1,2)$. As such, there appears to be minimal impact of SGLT2 inhibition upon macrovascular (arterial atheromatous) disease-but overall, despite experiencing the same frequency of cardiovascular events, survival nonetheless appears to be better in those taking SGLT2 inhibitors, a benefit that strikingly manifests within the first few months of treatment.

Cellular injury, necrosis, and programmed cell death (apoptosis, necroptosis, autophagy) are important pathophysiological features of a number of maladaptive processes in the heart, including myocardial ischemia and heart failure (6). We therefore hypothesized that despite a similar cardiovascular event rate from events such as acute myocardial ischemia, the improved cardiovascular survival arising from SGLT2 inhibition was through direct myocardial cytoprotection. In a rat, this can be tested in an experimental model of injurious ischemia/ reperfusion injury, whereby diabetic animals treated with an SGLT2 inhibitor would be anticipated to have smaller myocardial infarcts. Moreover, if the cardiovascular benefits of SGLT inhibitors are genuinely pleiotropic, we hypothesized that the benefits of SGLT2 inhibition would also be found in those without diabetes.

In designing our experiments, we observed that whereas the survival curves in the EMPA-REG and CANVAS trials separate quickly, it still takes weeks to see the survival curves diverge. As such, we undertook to treat both diabetic and nondiabetic rats for a period of 4 weeks. Moreover, because treatment with an SGLT2 inhibitor will invariably affect circulating blood glucose at the time of myocardial infarction in vivo, we harvested the hearts and undertook the 
FIGURE 1 Characterization of the ZL and ZDF Phenotypes

\section{A Body Mass Index (BMI) on the day of experiment
ZL vs ZDF}

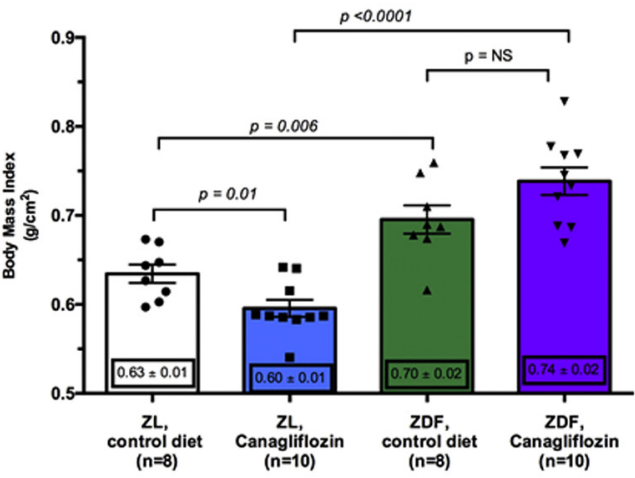

B

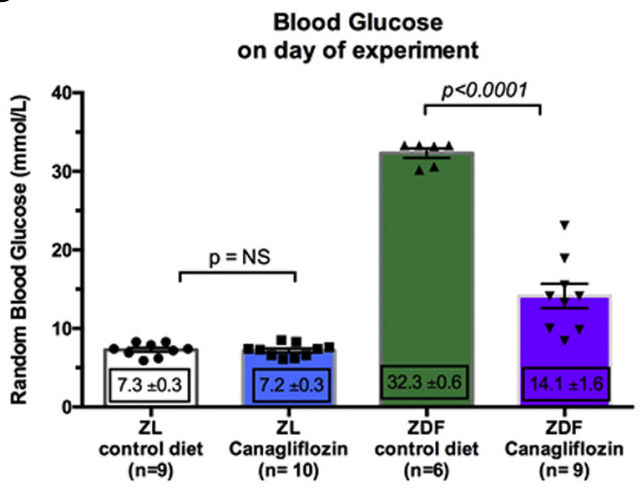

C

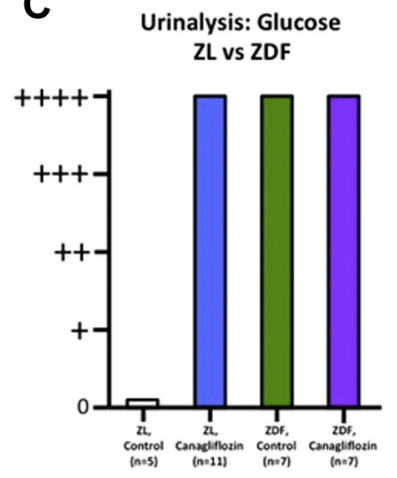

D

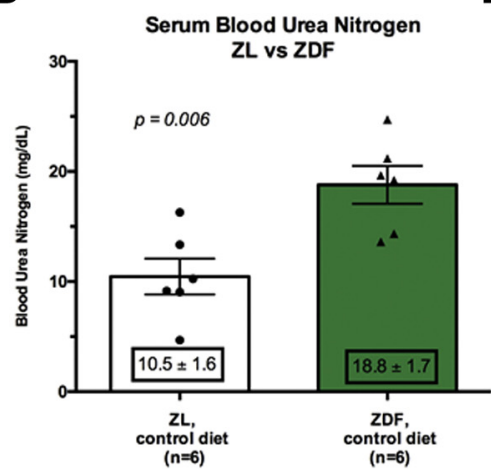

E

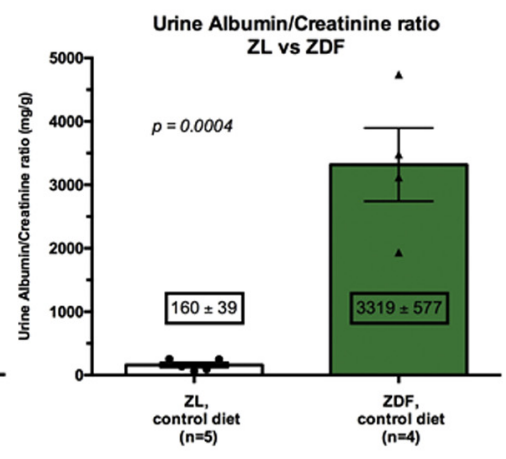

(A) Body weight index. The diabetic ZDF rats were significantly larger than the nondiabetic ZL rats. Canagliflozin administration in the ZL led to a significant reduction in body mass index that was absent in the ZDF diabetic rats. $n=8$ to 10 per group. (B) Random glucose concentration on day of experiment. As expected, ZDF diabetic rats had significantly higher blood glucose concentrations compared to the nondiabetic ZL controls ( $p<0.0001 ; n=6$ to 9 per group). Canagliflozin had no impact upon blood glucose in the $Z L$ group ( $p=N S ; n=9$ to 10 per group), but significantly reduced glucose in the diabetic ZDF rats ( $p<0.0001 ; n=6$ to 9 per group). (C to E) Renal manifestations of diabetes in the ZDF rats. (C) Urine glucose, measured by urinalysis strip test. No glucosuria was detectable in the control ZL rats, but there was significant glucosuria in ZL rats on canagliflozin. As expected, significant glucosuria was found in both ZDF control and canagliflozintreated groups. (D) Blood urea nitrogen was significantly higher in the ZDF rats compared with the nondiabetic ZL: $11 \pm 2 \mathrm{mg} / \mathrm{dl}$ versus $19 \pm 2 \mathrm{mg} / \mathrm{dl}$ ( $\mathrm{p}=0.006, \mathrm{n}=6$ per group). (E) A similar pattern was observed in the urine albumin/creatinine ratio-the diabetic ZDF rats demonstrating a significantly higher albumin excretion compared with the nondiabetic ZL rat: $160 \pm 39 \mathrm{mg} / \mathrm{g}$ versus 3,319 $\pm 577 \mathrm{mg} / \mathrm{g}$ ( $p=0.0004 ; n=4$ to 5 per group). ZDF = Zucker Diabetic Fatty; ZL = Zucker Lean.

experiments in an ex vivo Langendorff model, with perfused glucose concentration controlled in all experiments.

Finally, we wished to ascertain whether the SGLT2 inhibitor would have a direct, cardioprotective effect in the isolated heart, and to this end, we undertook a further group of experiments with "acute" exposure to the SGLT2 inhibitor, with the drug added to the Langendorff perfusate throughout the perfusion protocol.

Using the SGLT2 inhibitor, canagliflozin, in a reverse-translational study, we found that long-term pre-administration over 4 weeks led to a significant attenuation of myocardial infarct size in both diabetic Zucker Diabetic Fatty (ZDF) and nondiabetic Zucker Lean (ZL) rats. This observation may have significant impact for future translational studies in the repurposing of this new class of glucose-lowering drugs in all patients, irrespective of diabetic status, with highrisk cardiovascular disease.

\section{METHODS}

For a detailed description of all methods, see the Supplemental Appendix. In brief, ZL and ZDF rats were monitored weekly with random blood glucose 
FIGURE 2 Impact of Canagliflozin in Nondiabetic ZL and Diabetic ZDF Rats

A Canagliflozin pharmacodynamics (Glucose)
ZL vs ZDF
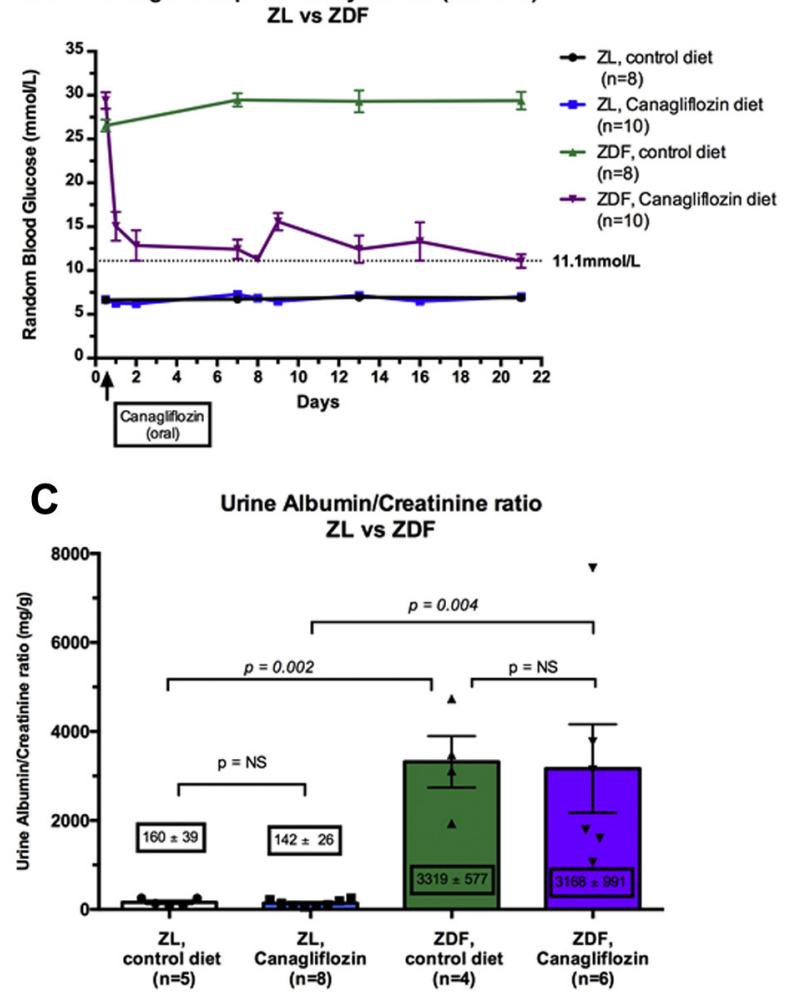

B
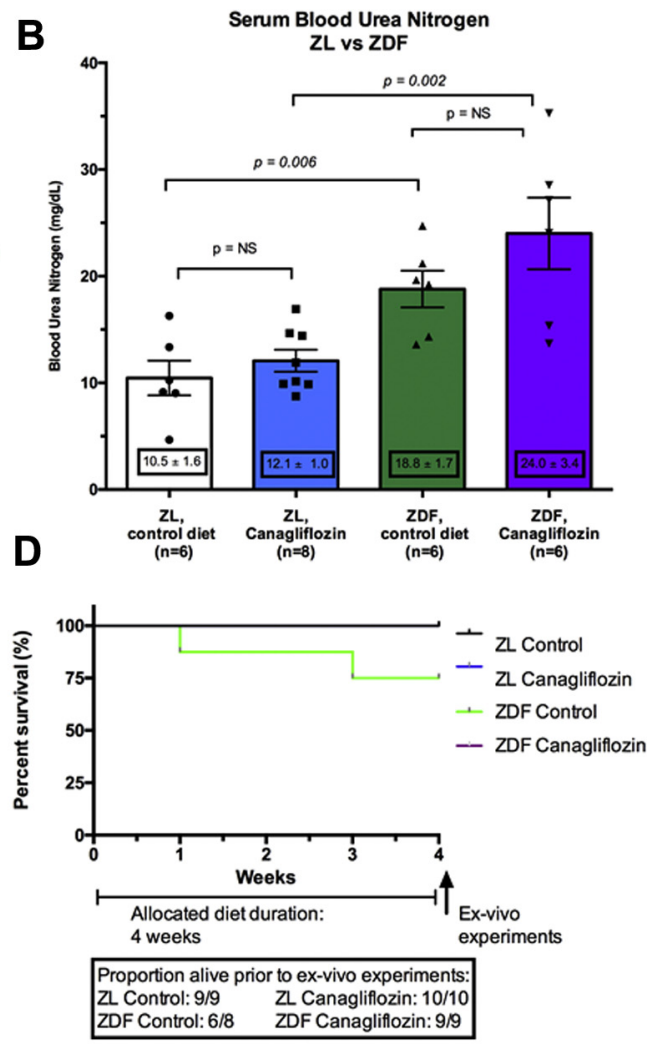

(A) Canagliflozin had a rapid and sustained impact upon circulating blood glucose in the diabetic ZDF rats compared with the untreated animals. By contrast, canagliflozin had no impact upon circulating blood glucose in the nondiabetic $Z \mathrm{~L}$ rats $(p=0.002 ; n=8$ to 10 per group). (B) After 4 week's treatment, canagliflozin had little impact upon blood urea nitrogen in either ZL or ZDF rats ( $p=N S$; $n=6$ to 8 per group). (C) As with BUN, there was little impact from 4-week oral canagliflozin administration in either ZL or ZDF rats upon albumin/ creatinine ratios ( $p=N S ; n=4$ to 8 per group). (D) Kaplan-Meier survival curve. Two animals, both in the control diabetic ZDF group, had to be euthanized for severe urinary sepsis. All other groups completed without events. Abbreviations as in Figure 1.

assessment, and fed either standard or high-fat chow, either fortified with canagliflozin or without (control) for a period of 4 weeks before harvesting the heart and Langendorff perfusion. All feeds, both with and without drug, were prepared by Research Diets (New Brunswick, New Jersey) based on the diet formulations provided by Janssen Research and Development (Springhouse, Pennsylvania). Using this formulation, the canagliflozin-fortified feed results in a circulating canagliflozin concentration ( $10 \mu \mathrm{mol} / \mathrm{l})$ equivalent to that found in human subjects taking maintenance canagliflozin, $300 \mathrm{mg}$ daily (7). Different diets were used for nondiabetic ZL and diabetic ZDF rats to account for the quantity of food eaten by these rats: the details of these feeds are detailed in the Supplemental Appendix.

Animals used for the acute administration of canagliflozin were nondiabetic Sprague-Dawley rats where canagliflozin (Janssen Research and Development) or vehicle, dimethyl sulfoxide (DMSO) (0.05\% DMSO, Sigma Aldrich, Poole, United Kingdom) was perfused throughout the Langendorff experiment.

RANDOMIZATION. All experiments were block randomized. Analysis was performed by 2 blind observers and arbitrated by a third independent adjudicator if required. Once all results were available, the data were unblinded and analyzed.

STATISTICAL ANALYSIS. All analyses were performed using GraphPad Prism version 6 (GraphPad Software, San Diego, California). The specific statistical test used is reported next to each result. An unpaired $t$-test was used for 2 independent groups of continuous variables and a 1-way analysis of variance with Tukey's multiple comparison test for 3 or more independent groups. Data are presented as mean \pm SEM. $\mathrm{N}$ values are either displayed in the 
FIGURE 3 A CONSORT-Style Diagram for Infarct Assessment in the 4-Week Administration Study

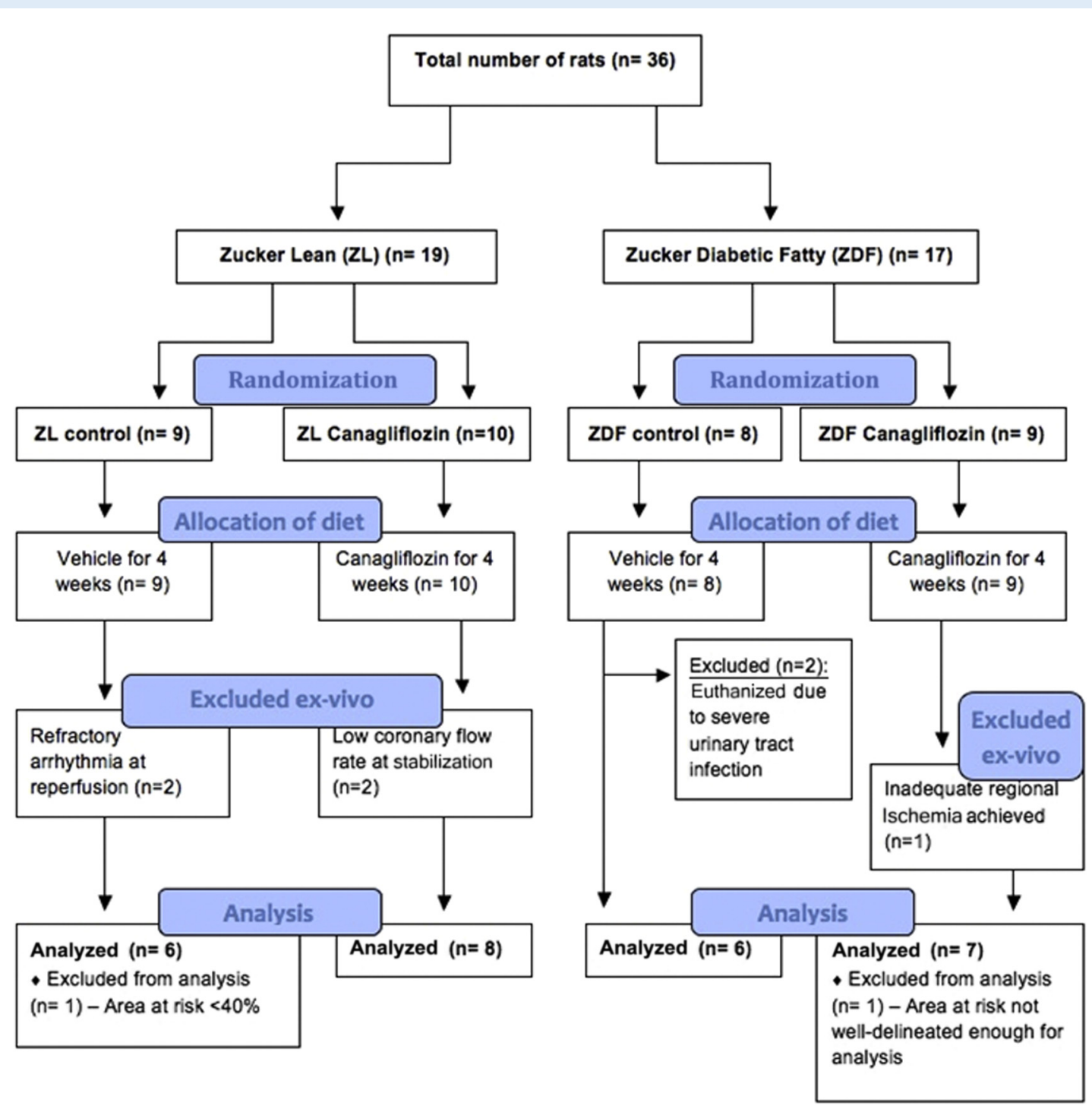

Thirty-six animals were started into the study, of which 29 completed through to analysis. Reasons for and timings of animal exclusions shown in all groups. Pre priori exclusion criteria are shown in the Supplemental Appendix. Abbreviations as in Figure 1.

figure or described in the figure legend for each experiment. A significance level of $5 \%(\alpha=0.05)$ and $80 \%$ power $(\beta=0.20)$ were used. Statistical significance was reported if $\mathrm{p}$ was $<0.05$ and results where p was $>0.05$ were reported as nonsignificant.

\section{RESULTS}

CHARACTERIZATION OF THE ZDF DIABETIC PHENOTYPE. To ensure that our ZDF rats represented a reasonable facsimile of the diabetic cohort represented within the EMPA-REG and CANVAS studies, we undertook characterization of the nondiabetic ZL and diabetic ZDF rats. We found, as expected, that the ZDF rats were obese and hyperglycemic (Figures $1 \mathrm{~A}$ and $1 \mathrm{~B}$ ) and hyperglucosuric (Figure 1C). In addition, the ZDF rats were found to have evidence of end-organ manifestations of their diabetes, as represented by abnormal renal function and albuminuria (Figures $1 \mathrm{C}$ and 1D). We are therefore confident that the ZDF represents a reasonable approximation of the human obese type 2 diabetic phenotype with significant and established diabetes at the time of experimentation.

Unexpectedly, we found that diabetic rats treated with canagliflozin were heavier than untreated 
diabetic rats; the expected weight loss from the calorific depletion associated with SGLT2-dependent glycosuria was, however, observed in the canagliflozin-treated nondiabetic ZL rats. Growth curves are shown in Supplemental Figure 1: the control-diet diabetic ZDF rats started heavier than the nondiabetic ZL rats, but failed to gain significant weight over the 4 weeks of feeding. By contrast, nondiabetic ZL rats gained weight in a linear fashion over the same 4-week period. Interestingly, the pattern and rate of weight gain seen in nondiabetic rats were mirrored in diabetic ZDF rats fed with canagliflozin, suggesting a healthier animal concomitant with better-controlled diabetes, an interpretation fitting with empirical observations of these animals' physical condition.

CHARACTERIZATION OF THE EFFICACY OF CANAGLIFLOZIN IN LOWERING CIRCULATING GLUCOSE. To ensure that oral administration of canagliflozin, via fortification of the chow, was an effective antihyperglycemic intervention in our rat model, we observed the random glucose profile in both nondiabetic ZL and diabetic ZDF rats throughout the treatment lead-in period. We found that canagliflozin was highly effective in lowering blood glucose concentrations in the ZDF rats within a short period from the onset of oral drug administration. Significantly improved blood glucose control was evident throughout the canagliflozin treatment course compared with control, with random blood glucose of $16 \pm 4 \mathrm{mmol} / \mathrm{l}$ versus $29 \pm 1 \mathrm{mmol} / \mathrm{l}$, respectively $(\mathrm{p}=$ 0.002) (Figure 2A).

Importantly, canagliflozin had no impact upon circulating glucose in the nondiabetic ZL rats, with equivalent blood glucose being recorded in both groups ( $\mathrm{p}=\mathrm{NS}$ ) (Figure 2A). Importantly, we found no evidence of hypoglycemia in either canagliflozin treatment group, despite the presence of significant glucosuria in the canagliflozin-treated nondiabetic ZL rats (Figure $1 \mathrm{C}$ ).

Interestingly, there was no attenuation of renal dysfunction in the diabetic canagliflozin-treated group ( $\mathrm{p}=\mathrm{NS}$ ) (Figures $2 \mathrm{~B}$ and $2 \mathrm{C}$ ). Unfortunately, our urinalysis assay saturates at glucose levels in excess of $110 \mathrm{mmol} / \mathrm{l}$, but higher urinary glucose would be anticipated in this group (Figure 1C). With respect to animal mortality, only 2 deaths were recorded-both animals were euthanized for severe urinary tract infection, and these events were found to occur only in animals in the untreated control diabetic ZDF group (Figures 2D and 3 ). The impact of diabetes and canagliflozin upon un-paced heart rate and liver: body weight ratio
FIGURE 4 Infarct Size Reduction Following Long-Term 4-Week Oral Administration of Canagliflozin

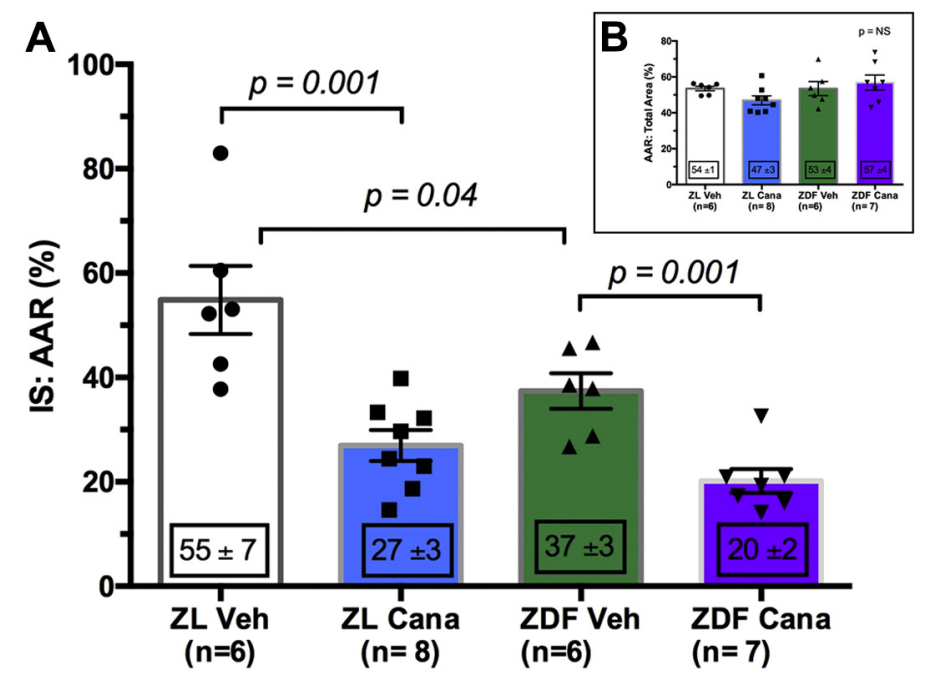

(A) In both diabetic ZDF and nondiabetic ZL rats, we found a significant reduction of infarct size compared with control. In nondiabetic rats, infarct size was reduced from $55 \pm 7 \%$ to $27 \pm 3 \%$ ( $p=0.001 ; n=6$ to 8 per group). In the diabetic ZDF rats, a similar reduction of infarct size was also observed with infarct size reducing from $37 \pm 3 \%$ to $20 \pm 2 \%$ ( $p=0.001 ; n=6$ to 8 ). There was a modest, but significant, difference in infarct size between control diet-treated $Z L$ and ZDF rats $(p=0.04)$. (B) Area at risk in all groups was equivalent ( $p=N S ; n=6$ to 8 per group). Cana = canagliflozin; IS:AAR = infarct size/area at risk ratio; ND = nonsignificant; Veh = vehicle; other abbreviations as in Figure 1.

are summarized in Supplemental Figures 3 and 4, respectively.

IMPACT OF 4-WEEK ORAL CANAGLIFLOZIN ON MYOCARDIAL INFARCT SIZE. For this investigation, we used 36 animals. Of these, 9 had to be excluded for reasons summarized in Figure 3. Twenty-seven animals completed the full experimental protocol.

We found a small, but significant, difference between myocardial infarct size in the control arms of the diabetic ZDF and the nondiabetic ZL rat heart groups ( $\mathrm{p}=0.04$ ) (Figure 4A, Supplemental Figure 2). This difference is expected in Langendorff-perfused hearts where glucose is the sole energy substrate (see review [8]). We found that canagliflozin, mirroring the important data by Andreadou et al. (9) in the mouse, significantly reduced myocardial infarct size in diabetic ZDF rats. Infarct size relative to the control chow-fed ZDF rats was significantly attenuated, from $37 \pm 3 \%$ to $20 \pm 2 \%$ of the area at risk ( $\mathrm{p}=0.001$ ) (Figure 4A). Importantly, canagliflozin also significantly abrogated myocardial injury in the nondiabetic ZL rats, reducing infarct size from 
FIGURE 5 Infarct Sizes Following Short-Term Ex-Vivo Administration in Nondiabetic Sprague-Dawley Rats

A

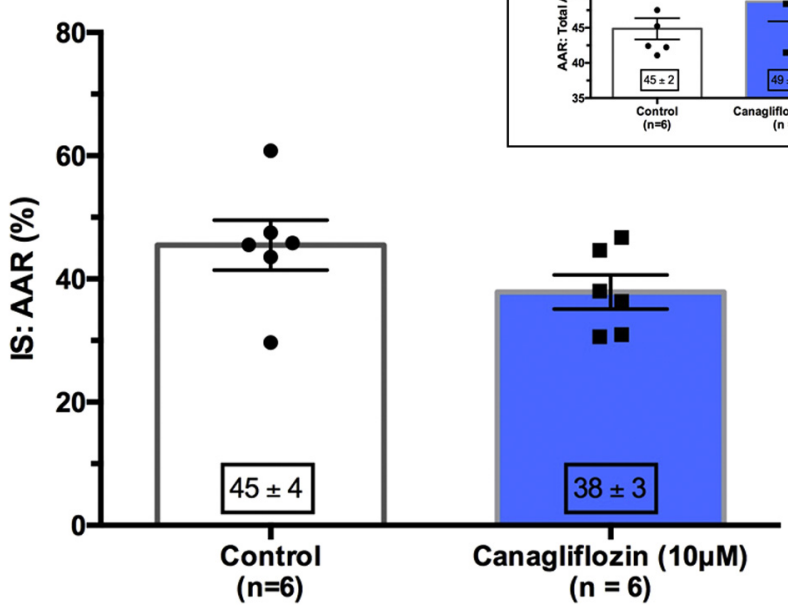

(A) In contrast to the cardioprotective effect of 4-week oral administration of canagliflozin, we found no evidence of infarct reduction with short-term, ex-vivo administration of canagliflozin: infarct sizes of $45 \pm 4 \%$ versus $38 \pm 3 \%$ ( $p=N S, n=6$ per group) in the vehicle control group. (B) There was no difference in the area at risk in either of the treatment groups (DMSO vehicle control versus canagliflozin; $\mathrm{p}=\mathrm{NS} ; \mathrm{n}=6$ per group). DMSO = dimethyl sulfoxide; other abbreviations as in Figures 1 and 4.
$55 \pm 7 \%$ to $27 \pm 3 \%(\mathrm{p}=0.001)$ (Figure $4 \mathrm{~A})$. The areas at risk in all control and treatment groups were similar with no statistical difference (Figure 4B). The impact of canagliflozin upon coronary flow and left ventricular developed pressure are summarized in Supplemental Figures 5 and 6, respectively.

\section{EFFECT OF SHORT-TERM ADMINISTRATION} OF CANAGLIFLOZIN AT THE TIME OF ISCHEMIA/REPERFUSION INJURY. TO ascertain whether acute administration of canagliflozin is protective against injurious ischemia/reperfusion injury in the nondiabetic rat, we subjected the isolated Sprague-Dawley rat heart to ischemia/reperfusion injury in the presence of vehicle (0.05\% DMSO) or 10 $\mu \mathrm{mol} / \mathrm{l}$ canagliflozin throughout the perfusion protocol (during 40-min stabilization, 35-min regional ischemia, and throughout the $2 \mathrm{~h}$ of reperfusion). The concentration used is equivalent to the plasma concentration of canagliflozin in diet-fed ZDF rats (7). Baseline characteristics were identical between groups: both demonstrating a nondiabetic level of random blood glucose and identical anthropological measurements between groups (Supplemental Table 1). No rats had to be excluded from this study, and all rat data were included in the final analysis.
Of note, short-term, ex-vivo canagliflozin failed to significantly alter infarct size, with treatment versus control of $38 \pm 3 \%$ versus $45 \pm 4 \%$, respectively ( $\mathrm{p}=$ 0.15 ) (Figure 5A). There was no difference in the area at risk between any of the groups (Figure 5B).

\section{DISCUSSION}

Our study provides the first evidence to our knowledge that long-term oral administration of canagliflozin over a period of 4 weeks is cardioprotective, ameliorating myocardial infarct size in both diabetic and nondiabetic rats, independent of glucose concentration at the time of ischemia/reperfusion injury. The latter observation, that canagliflozin-induced protection in the nondiabetic rat, is particularly noteworthy: a clinically available SGLT2 inhibitor, canagliflozin, appears to have a cardiovascular and cardioprotective role that extends beyond (and probably also independent of) its intended indication in the management of hyperglycemia in type 2 diabetes mellitus.

\section{LONG-TERM ORAL CANAGLIFLOZIN ATTENUATES} MYOCARDIAL INFARCTION IN THE DIABETIC RAT. In the diabetic ZDF rats, attenuating the extent of myocardial necrosis hints towards a novel mechanism underlying the significant reduction of cardiovascular mortality found in the clinical outcome studies, EMPA-REG and CANVAS $(1,2)$. Although the clinical data reveal no evidence that SGLT2 inhibitors reduce the number of cardiovascular events such as acute coronary syndromes, they may reduce the myocardial injury that occurs as a consequence of these events. A reduction of myocardial necrosis may thus improve both the immediate and long-term survivability of acute myocardial infarction and reduce the progression into ischemic cardiomyopathy and heart failure-a hypothesis that warrants further investigation.

Interestingly, the protection from long-term ingestion of canagliflozin was found in hearts that were removed and perfused, ex vivo, with a perfusate that contained a fixed concentration of glucose (11 mmol/l). We designed the experiments this way intentionally to avoid potential confounding the effects of glucose-lowering by canagliflozin at the time of ischemia/reperfusion injury. Moreover, Langendorff perfusion removes, through washout, other metabolic substrates that may confound canagliflozin administration (e.g., hepatic generation of ketones (10), as discussed further later in the text) are excluded as a potential mechanism of cardioprotection. Moreover, that these explanted hearts were protected, despite $40 \mathrm{~min}$ of crystalloid 
washout before ischemia, suggests a mechanism that imbues a "memory," potentially through the recruitment of signaling pathways. And if a signaling pathway, it is a pathway whose efficacy, unlike that of ischemic conditioning (11), is seemingly not affected by the presence of significant diabetes (the severity of the diabetic phenotype confirmed by evidence of the development of nephropathy). One such mechanism may be through a Jak-STAT3 pathway, as suggested by Iliodromitis's group (9)-but there may be others.

LONG-TERM ORAL CANAGLIFLOZIN ATTENUATES MYOCARDIAL INFARCTION IN THE NONDIABETIC RAT. Although the observation that canagliflozin attenuates infarct size in the diabetic rat is important, the principal novelty in this study comes from our data in the nondiabetic group of animals. We observe that long-term oral canagliflozin administration significantly reduces myocardial infarct size in the nondiabetic ZL rat heart. These data have 3 provocative implications:

1. The potentially paradigm-shifting observation that SGLT2 inhibitors may be repurposed for the management of high-risk nondiabetic patients with significant pre-existing cardiovascular disease.

2. Canagliflozin is not a pure diabetic drug, and possesses pleiotropic effects that extend beyond purely lowering serum glucose.

3. The cardioprotective effect of canagliflozin is only manifest when administered orally over a period of weeks, which challenges current thinking in terms of mechanisms that appear to extend beyond a direct effect upon either the myocardium or kidney.

EX-VIVO CANAGLIFLOZIN FAILS TO PROTECT THE NONDIABETIC RAT HEART. In contrast to the longterm oral administration, the short-term administration of canagliflozin, ex vivo, administered at a concentration of $10 \mu \mathrm{mol} / \mathrm{l}$ (equivalent to the circulating concentration in patients taking canagliflozin, $300 \mathrm{mg}$ once daily [7]) throughout the perfusion protocol, failed to reduce infarct size. This concentration of canagliflozin is also equivalent to a rat steady-state circulating serum canagliflozin concentration from oral digestion of drug, and a concentration that is sufficient to inhibit both SGLT2 and SGLT1, but insufficient to abrogate GLUT (glucose transporter) activity (12). The observation that short-term ex vivo administration of canagliflozin fails to protect the isolated heart may provide some further clues to the potential mechanism of action, because it appears to preclude a direct-acting cardioprotective effect of the drug upon the myocardium. Administering the drug ex vivo removes any confounding endocrine effects that the drug might elicit from any other organ system in vivo, as might occur in our long-term administration model. Thus, in the absence of infarct attenuation from ex vivo administration of canagliflozin, it would appear that the cardioprotective effect of SGLT inhibition is unlikely to be through the drug acting directly upon the myocardium itself and hints toward an endocrine (and downstream signaling) or metabolic effect to explain the beneficial effect of long-term oral administration of canagliflozin. However, our data appear not to support a metabolic effect: in our long-term canagliflozin model, the protection was seen ex vivo with a sole metabolic substrate: glucose at a concentration of 11 $\mathrm{mmol} / \mathrm{l}$. This makes preferential energy-substrate switching, as proposed in the ketone hypothesis (10), unlikely as an explanation for the cardioprotection observed. Following explantation and Langendorff perfusion of the heart, ketones will be rapidly washed out of the coronary circulation because the crystalloid-perfused Langendorff model is associated with far higher coronary flows than found in vivo (13). Thus, ketones will rapidly fall to negligible levels within the myocardium, and are unlikely to supplant the plentiful supply of glucose as the heart's primary fuel source in the Langendorff perfused model. Of course, we have not excluded the role of endogenous myocardial glycogenesis, but interestingly, long-term SGLT2 inhibition leads to diminution of kidney and liver glycogen stores (14). The role of glycogen in myocardial ischemia reperfusion injury is complex-canonical succinate synthesis through gluconeogenesis during myocardial ischemia is likely beneficial, but potentially deleterious during reperfusion through reversal of complex II of the mitochondrial transport chain (15). The impact of glycogen depletion on myocardial injury would be interesting to study further.

The sodium hydrogen exchange (NHE) hypothesis appeared to be a strong and attractive contender to explain the cardioprotection in our long-term canagliflozin administration studies $(16,17)$. Previous investigations using cariporide and amiloride in animal models reveal highly efficacious anti-ischemic benefits of NHE inhibition against myocardial infarction, particularly when administered before the onset of myocardial ischemia (18-21). Thus, we had anticipated the short-term ex vivo study to provide further evidence of infarct size limitation. Indeed, in the excellent study from Zuurbier's group (17), with 3 $\mu \mathrm{mol} / \mathrm{l}$ canagliflozin, they demonstrated highly effective attenuation of NHE activity. Given the 
similarity in concentration of canagliflozin in our and in Zuurbier's cell-based model, we were surprised that we found no protection in our ex-vivo model. Might the protective effects of long-term administration of canagliflozin be mediated through NHE inhibition? Encouragingly, protection was observed in both diabetic and nondiabetic animals as expected. However, with 40 min of washout before induction of ischemia, it seems somewhat unlikely that significant quantities of canagliflozin would remain within the heart. Our data would therefore appear to suggest that the observed protection from long-term administration of canagliflozin is less likely to be mediated through NHE inhibition, but perhaps through another pleiotropic pathway capable of triggering a "memory" effect through activation of signaling cascades. Already identified candidate pathways include the aforementioned Jak/STAT3 pathway (9) that may also help attenuate oxidative stress and fibrotic myocardial remodeling (22) or perhaps through AMPK (23) (also found in kidney to reduce ischemia/reperfusion injury [24]), although these are not hypotheses that we have yet tested.

Finally, SGLT2 inhibitors have been found to imbue significant protection in the vasculature of diabetic ZDF rats, with preservation of endothelial function. This endothelial protection appears to be mediated through attenuation of long-term glucotoxicity and amelioration of oxidative stress (25). This could translate into myocardial protection ex vivo, but we did not find significant differences in coronary flow in our model between canagliflozin-treated versus control-treated animals (data shown in the Supplemental Appendix). Moreover, if the protection were mediated primarily as a mechanism designed to abrogate glucotoxicity, this hypothesis fails to explain why canagliflozin protects the nondiabetic heart. However, it would be interesting to repeat these experiments in the nondiabetic ZL rat to see whether the cytoprotective phenotype is evident in the absence of injurious elevated blood glucose.

\section{CANAGLIFLOZIN-MEDIATED CARDIOPROTECTION} APPEARS INDEPENDENT OF CIRCULATING GLUCOSE. As expected, we found canagliflozin to be highly effective at reducing circulating blood glucose in our diabetic rat model. Although we did not see the random blood glucose level in canagliflozintreated diabetic ZDF rats fall into the nondiabetic range, the drug was nonetheless still highly effective at reducing infarct size, suggesting that complete restoration of random blood glucose into the "normal" nondiabetic range is unnecessary to imbue the cardioprotection observed. Moreover, canagliflozin failed to have an impact on circulating blood glucose levels in the nondiabetic animals: random glucose levels were identical in both nondiabetic control and canagliflozin-treated rats. There are 2 observations in respect to this data: 1) that canagliflozin can be administered to nondiabetic animals without fear of triggering potentially injurious hypoglycemia; and 2) that lowering blood glucose is not a prerequisite for attenuation of myocardial infarct size. Therefore, glucose lowering in the diabetic ZDF animals is a good biomarker of canagliflozin-mediated SGLT2 inhibition, but the in vivo lowering of glucose is not conditional for the triggering of infarct-size reduction when the heart is explanted and perfused ex vivo. Furthermore, as alluded to above, as the hearts were maintained with a perfused glucose concentration of $11 \mathrm{mmol} / \mathrm{l}$ throughout perfusion, any confounding effect of differences in circulating glucose concentration is effectively removed from our experiment.

Finally, it is also interesting to observe that long-term oral canagliflozin is equally protective in both nondiabetic and diabetic animals. This contrasts with the majority of cardioprotective interventions whose efficacy is blunted in the presence of the diabetic phenotype (11). This, therefore, leads us to speculate that the mechanisms of protection are different from, and potentially additive to, more established experimental models of myocardial protection, such as ischemic or pharmacological conditioning. If this were to be the case, then it offers the opportunity to augment myocardial protection through combined therapeutic approaches at the time of presentation of an acute coronary syndrome, to optimize patient outcome.

ABSENCE OF RENOPRESERVATION. In establishing our diabetic model, we wanted to determine the severity of the diabetic phenotype. The SGLT2 outcome studies have all been performed in models of established type 2 diabetes mellitus, and typically in patients with high cardiovascular risk. We therefore wanted to ascertain whether our model displayed characteristics of diabetic end-organ damage in the form of albuminuria. Our diabetic ZDF rats did indeed display evidence of significant albuminuria at the point at which the hearts were harvested for ex vivo Langendorff perfusion. The lack of any meaningful difference between the canagliflozin-fed and control ZDF rats is not, however, unexpected. The renoprotective effects of SGLT2 inhibition typically take many months to manifest $(2,26)$, which contrasts with the comparatively rapid separation of the cardiovascular outcome curves. We designed our study primarily as an investigation into cardioprotection; a study with renoprotection as a 
primary endpoint would likely mandate a much longer duration of drug treatment.

DIABETIC COMPLICATIONS. It was initially surprising that the only serious, life-threatening complication found during our long-term study was infective. As might have been anticipated, the source of infection was, in both cases, urinary tract. However, these 2 events were in the nontreated control diabetic ZDF rats and not in animals treated with canagliflozin. In total, 2 animals in the control ZDF group had to be euthanized for serious sepsis; neither of the canagliflozin-treated groups (diabetic or nondiabetic) had evidence of septic complications. Both diabetic ZDF groups had significant glycosuria, whereas the untreated control ZDF also had significant hyperglycemia. The sepsis, therefore, is much more likely to be secondary to the uncontrolled diabetes in the control animals, whereas the infective risk associated with canagliflozin-induced glucosuria was easily managed by simple animal husbandry and hygiene methods. No animal deaths were found related to cardiovascular causes, but our study was not powered for this endpoint, nor was it run for a sufficient period for such complications to become manifest.

STUDY LIMITATIONS. In designing our studies, we accepted a number of compromises. To avoid the confusion that may ensue with polypharmacy, we did not treat the control diabetic animals to manage their hyperglycemia. These animals displayed high levels of glycemia, and 2 animals had septic complications that were rapidly identified and managed. We therefore feel that prolonging the duration of study beyond 4 weeks as designed would not have been feasible. However, the infarct size data are compelling: administering canagliflozin, irrespective of diabetic status, resulted in a pronounced reduction of myocardial infarct size.

As all diabetic patients in the clinical outcome studies were undertaken in the presence of antihyperglycemic agents, a future study may be constructed at the outset to include diabetic animals managed with metformin, the backbone of contemporary type 2 diabetic management. Indeed, this may well be mandated in any future study designed to look at cardiovascular complications and renal outcomes where much longer treatment periods would need to be considered.

We do not believe that the severity of the diabetes had an adverse impact upon the outcome of our study; in fact, the infarct size of the diabetic animals was entirely in line with previous short-term studies in other diabetic models (such as streptozocin-treated or Goto-Kakizaki lean diabetic rats) and from our own group and others $(27,28)$. However, having established that canagliflozin is cardioprotective, it would be useful to demonstrate that this protective phenotype is reproducible on top of existing strategies for managing elevated blood sugar.

Interestingly, it is well recognized that diabetic hearts, when Langendorff-perfused with glucose as the sole substrate, will have a smaller infarct size compared with the nondiabetic heart under the same conditions (see review [8]). Although a reductionist approach in metabolic substrate provision has its limitations, there are advantages in that we have excluded other potential metabolic substrates that have been postulated (such as ketone bodies). From our data, future more in-depth analysis of the myocardial metabolome may be undertaken, and for example, the impact of any glycogen depletion that may result from long-term SGLT2 inhibition, investigated.

Finally, our short-term canagliflozin study was performed in Sprague Dawley rats, rather than the ZL strain. Neither strain of rat are diabetic. Both strains reveal similar infarct sizes when subjected to $35 \mathrm{~min}$ of regional ischemia and $2 \mathrm{~h}$ reperfusion. Although there are differences between individual strains of murine and rat models, and their sensitivity to myocardial ischemia/reperfusion injury, given baseline similarities in infarct size, we would have expected canagliflozin to be as protective in Sprague Dawley rats as the ZL. The absence of protection observed is, therefore, informative, but minor strain differences cannot be completely excluded.

\section{CONCLUSIONS}

We demonstrate that long-term oral administration of canagliflozin results in significant reduction in myocardial infarct size, irrespective of glucose lowering or the presence of diabetes. This protection appears not to be mediated via a direct effect of canagliflozin upon the myocardium, but via an intermediate signaling mechanism that has yet to be identified. Our study, therefore, provides new insights into the potential cardiovascular benefits of SGLT2 inhibition and even points to a potential and important translational repurposing of these drugs to reduce cardiovascular mortality in nondiabetic patients.

ADDRESS FOR CORRESPONDENCE: Prof. Derek M. Yellon, The Hatter Cardiovascular Institute, University College London, 67 Chenies Mews, London WC1E 6HX, United Kingdom. E-mail: d.yellon@ucl.ac.uk. 


\section{PERSPECTIVES}

COMPETENCY IN MEDICAL KNOWLEDGE: SGLT2 inhibitors are known to improve cardiovascular outcomes in high-risk diabetic patients. We demonstrate for the first time that SGLT2 inhibitors attenuate infarct size in both diabetic and nondiabetic rats. This class of antihyperglycemic drug, therefore, appears to have cardioprotective properties that extend beyond their ability to lower circulating blood glucose.

TRANSLATIONAL OUTLOOK 1: Long-term SGLT2 inhibition is cardioprotective, reducing myocardial infarct size following injurious myocardial ischemia. This is a favorable characteristic for a diabetic therapy, supporting their use in diabetic patients with high risk of, or established, cardiovascular disease.

TRANSLATIONAL OUTLOOK 2: Our data suggest that infarct limitation is also seen in nondiabetic animals, raising the tantalizing potential for repurposing these drugs to improve cardiovascular outcomes in all high-risk cardiovascular patients, irrespective of diabetic status.

\section{REFERENCES}

1. Zinman B, Wanner C, Lachin JM, et al. Empagliflozin, cardiovascular outcomes, and mortality in type 2 diabetes. N Engl J Med 2015;373:2117-28.

2. Neal B, Perkovic V, Mahaffey KW, et al. Canagliflozin and cardiovascular and renal events in type 2 diabetes. N Engl J Med 2017;377:644-57.

3. Pioli MR, Ritter AMV, Modolo R. Unsweetening the heart: possible pleiotropic effects of SGLT2 inhibitors on cardio and cerebrovascular alterations in resistant hypertensive subjects. Am J Hypertens 2018;31:274-80.

4. Bell RM, Yellon DM. SGLT2 inhibitors: hypotheses on the mechanism of cardiovascular protection. Lancet Diabetes Endocrinol 2018;6:435-7.

5. Ahmed HM, Khraishah H, Cho L. Cardioprotective anti-hyperglycemic medications: a review of clinical trials. Eur Heart J 2018;39:2368-75.

6. Moe GW, Marin-Garcia J. Role of cell death in the progression of heart failure. Heart Fail Rev 2016;21:157-67.

7. Devineni $D$, Curtin $C R$, Polidori $D$, et al. Pharmacokinetics and pharmacodynamics of canagliflozin, a sodium glucose co-transporter 2 inhibitor in subjects with type 2 diabetes mellitus. J Clin Pharmacol 2013;53:601-10.

8. Whittington HJ, Babu GG, Mocanu MM, Yellon DM, Hausenloy DJ. The diabetic heart: too sweet for its own good? Cardiol Res Pract 2012 2012:845698.

9. Andreadou I, Efentakis $P$, Balafas $E$, et al. Empagliflozin limits myocardial infarction in vivo and cell death in vitro: role of STAT3, mitochondria, and redox aspects. Front Physiol 2017;8:1077.

10. Mudaliar S, Alloju S, Henry RR. Can a shift in fuel energetics explain the beneficial cardiorenal outcomes in the EMPA-REG OUTCOME study? A unifying hypothesis. Diabetes Care 2016;39: 1115-22.

11. Sack MN, Murphy E. The role of comorbidities in cardioprotection. J Cardiovasc Pharmacol Ther 2011;16:267-72.

12. Kuriyama C, Xu JZ, Lee SP, et al. Analysis of the effect of canagliflozinon renal glucose reabsorption and progression of hyperglycemia in Zucker diabetic Fatty rats. J Pharmacol Exp Ther 2014; 351:423-31.

13. Sutherland FJ, Hearse DJ. The isolated blood and perfusion fluid perfused heart. Pharmacol Res 2000;41:613-27.

14. Atageldiyeva $K$, Fujita $Y$, Yanagimachi $T$, et al. Sodium-glucose cotransporter 2 inhibitor and low carbohydrate diet affect gluconeogenesis and glycogen content differently in the kidney and the liver of non-diabetic mice. PLoS One 2016;11: e0157672.

15. Zhang J, Wang YT, Miller JH, Day MM Munger JC, Brookes PS. Accumulation of succinate in cardiac ischemia primarily occurs via canonical Krebs cycle activity. Cell Rep 2018;23: 2617-28.

16. Baartscheer $A$, Schumacher $C A$, Wust RC, et al Empagliflozin decreases myocardial cytoplasmic $\mathrm{Na}(+)$ through inhibition of the cardiac $\mathrm{Na}(+) /$ $\mathrm{H}(+)$ exchanger in rats and rabbits. Diabetologia 2017;60:568-73.

17. Uthman $L$, Baartscheer $A$, Bleijlevens $B$, et al. Class effects of SGLT2 inhibitors in mouse cardiomyocytes and hearts: inhibition of $\mathrm{Na}(+) / \mathrm{H}(+)$ exchanger, lowering of cytosolic $\mathrm{Na}(+)$ and vasodilation. Diabetologia 2018;61:722-6.

18. Avkiran $\mathrm{M}$, Marber MS. $\mathrm{Na}(+) / \mathrm{H}(+)$ exchange inhibitors for cardioprotective therapy: progress, problems and prospects. J Am Coll Cardiol 2002; 39:747-53.

19. Klein $H H$, Pich $S$, Bohle RM, Lindert Heimberg S, Nebendahl $\mathrm{K} . \mathrm{Na}(+) / \mathrm{H}(+)$ exchange inhibitor cariporide attenuates cell injury predominantly during ischemia and not at onset of reperfusion in porcine hearts with low residual blood flow. Circulation 2000;102:1977-82.

20. Hale SL, Kloner RA. Effect of combined K(ATP) channel activation and $\mathrm{Na}(+) / \mathrm{H}(+)$ exchange inhibition on infarct size in rabbits. Am J Physiol Heart Circ Physiol 2000;279:H2673-7.

21. Mirkovic S, Seymour AM, Fenning $A$, et al. Attenuation of cardiac fibrosis by pirfenidone and amiloride in DOCA-salt hypertensive rats. $\mathrm{Br}$ Pharmacol 2002:135:961-8.
22. Lee TM, Chang NC, Lin SZ. Dapagliflozin, a selective SGLT2 Inhibitor, attenuated cardiac fibrosis by regulating the macrophage polarization via STAT3 signaling in infarcted rat hearts. Free Radic Biol Med 2017;104:298-310.

23. Hawley SA, Ford RJ, Smith BK, et al. The $\mathrm{Na}+$ / glucose cotransporter inhibitor canagliflozin activates AMPK by inhibiting mitochondrial function and increasing cellular AMP levels. Diabetes 2016; 65:2784-94.

24. Chang YK, Choi H, Jeong JY, et al. Dapagliflozin, SGLT2 inhibitor, attenuates renal ischemiareperfusion injury. PLoS One 2016;11:e0158810.

25. Steven $S$, Oelze M, Hanf A, et al. The SGLT2 inhibitor empagliflozin improves the primary diabetic complications in ZDF rats. Redox Biol 2017; 13:370-85.

26. Wanner C, Inzucchi SE, Lachin JM, et al. Empagliflozin and progression of kidney disease in type 2 diabetes. N Engl J Med 2016;375:323-34.

27. Whittington $\mathrm{HJ}$, Harding I, Stephenson $\mathrm{Cl}$, et al. Cardioprotection in the aging, diabetic heart: the loss of protective Akt signalling. Cardiovasc Res 2013:99:694-704.

28. Korkmaz-Icoz S, Lehner A, Li S, et al. Mild type 2 diabetes mellitus reduces the susceptibility of the heart to ischemia/reperfusion injury: identification of underlying gene expression changes. J Diabetes Res 2015;2015:396414.

KEY WORDS cardioprotection, diabetes, ischemia-reperfusion injury, myocardial infarction, SGLT2 inhibitor

APPENDIX For an expanded Methods section as well as supplemental figures and tables, please see the online version of this paper.

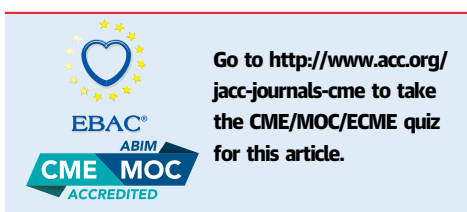

